\title{
Adaptive sampling in convergent beams
}

\author{
Julián Espinosa, David Mas, * Jorge Pérez, and Carlos Illueca \\ Departamento Óptica, Farmacología y Anatomía, Universidad de Alicante, P.O. Box 99, 03080 Alicante, Spain \\ *Corresponding author: david.mas@ua.es
}

Received June 4, 2008; revised July 14, 2008; accepted July 18, 2008; posted July 23, 2008 (Doc. ID 97056); published August 22, 2008

Numerical calculation of convergent Fresnel patterns through fast Fourier transform usually requires a large number of samples to fulfill the Nyquist sampling condition around the focus. From polynomial decomposition of the wavefront it is possible to determine which polynomial orders are the main contributors to the number of samples. This information can be used to properly modify the initial wavefront and relax the Nyquist condition thus giving a more efficient numerical algorithm. (c) 2008 Optical Society of America OCIS codes: $070.2025,200.4740$.

Numerical calculation of convergent Fresnel patterns through fast Fourier transform (FFT) usually requires a large number of samples $\left(4 \times 10^{6}\right)$ to fulfill the Nyquist condition around the focus [1]. Since the energy of a convergent beam becomes more concentrated as it propagates, it is expected that initial sampling conditions will be less restrictive as the pattern is nearer to the focus. In [2] the authors impose a sampling condition to the input signal and calculate the validity limit of some Fresnel through FFT algorithms that maintain a constant number of samples throughout the propagated distance. The main problem that arises with such an approach is that as light approaches the focus the energy becomes more concentrated, but the sampling rate remains constant (spectrum propagation algorithm) or diminishes with distance (direct and fractional Fourier transform methods) [2,3]. In this Letter, an alternative method is established; the object phase function is decomposed in polynomials. Separate analysis and manipulation of some terms permit decreasing the number of samples and thus faster and more efficient algorithms.

Consider the one-dimensional (1D) Fresnel pattern produced by an object $u_{0}$ at a distance $z$ when it is illuminated by a monochromatic plane wave with wavelength $\lambda$. Numerical calculations of the Fresnel integral can be done, if $\Delta x_{0} \Delta x_{z}=\lambda z N$ is fulfilled, as [2]

$$
\begin{aligned}
\left(u_{z}\right)_{\mu^{\prime}}= & \exp \left(i \pi \frac{\lambda z}{\Delta x_{0}^{2}} \mu^{\prime 2}\right) \mathrm{DFT} \\
& \times\left[u_{0}\left(\frac{\mu \Delta x_{0}}{N}\right) \exp \left(\frac{i \pi \Delta x_{0}^{2}}{\lambda z N^{2}} \mu^{2}\right)\right],
\end{aligned}
$$

where $\Delta x_{0}$ is the object size, $\Delta x_{z}$ is the sampling extension in the Fresnel domain, $N$ is the number of samples in both the object and the Fresnel domain, DFT is the discrete Fourier transform, and $\mu$ and $\mu^{\prime}$ are the integer sampling indexes. Nyquist condition $N \geqslant \Delta x_{0}^{2} /(\lambda z)$ obtained for this expression imposes an excessive number of sample points for very short focusing distances. Let us consider a generic phase function in our system, $W\left(x_{0}\right)$, so that the transmittance function can be expressed in a discrete form as

$$
u_{0}\left(\mu \frac{\Delta x_{0}}{N}\right)=\exp \left[i \frac{2 \pi}{\lambda} W\left(\mu \frac{\Delta x_{0}}{N}\right)\right] .
$$

The phase function can be expanded into a sum of polynomials. The general form of one term of the transmittance expansion, in both continuous and discrete forms, is

$$
\exp \left(i \frac{2 \pi}{\lambda} \beta_{n} x_{0}^{n}\right) \rightarrow \exp \left(i \frac{2 \pi}{\lambda} \beta_{n} \mu^{n} \frac{\left(\Delta x_{0}\right)^{n}}{N_{n}^{n}}\right),
$$

where $\beta_{n}$ and $n$ stand for the coefficient and the order of each expansion term. Thus, the phase function is equivalent to a sum of polynomials or, equivalently, to a product of phase factors, each one providing a different sampling requirement. The most restrictive condition obtained for correct sampling of all the phase factors will determine the number of samples needed for a correct reconstruction of object function, i.e.,

$$
N=\max \left(N_{n}\right), \quad N_{n} \geqslant \frac{n\left|\beta_{n}\right|\left(\Delta x_{0}\right)^{n}}{\lambda 2^{n-2}} .
$$

For a convergent phase, the expansion term is of the form $\exp \left[-i \pi \Delta x_{0}^{2} /\left(\lambda z_{c}\right)\right]$, so the coefficient and the Nyquist condition are $\beta_{2}=-1 /\left(2 z_{c}\right)$ and $N_{2} \geqslant \Delta x_{0}^{2} / \lambda z_{c}$, with $z_{c}$ being the convergence distance. In the case of phase functions with no dominant high-order terms, such as normal healthy human eye wavefront aberrations, the Nyquist condition is determined by the convergence term $\beta_{n}$. Coefficient values usually decrease with an increasing order and their progression compensate the effect of the increasing term $\left(\Delta x_{0}\right)^{n}$ in Eq. (4). Thus, first-order aberrations usually impose the most restrictive condition of the number of samples, that being the convergence term, the main one, as it will be later shown in Table 1 .

The generalization to the two-dimensional (2D) case is not evident owing to the existence of crossed terms. In many applications it is of special interest to decompose the phase function into radial Zernike polynomials, $Z_{n, m}(\rho, \theta)=\mathrm{N}_{n, m} R_{n, m}(\rho) M_{m}(\theta)$, in polar coordinates $(\rho, \theta)$ owing to the fact that this way there are no crossed dimensions, and the terms have a degree of radial symmetry since they are periodic in $\theta$ : 
Table 1. Sampling Requirements for Each $G_{j}$ Polynomial for (A) Spherical Wavefront $z_{c}=15.75 \mathrm{~mm}$, (B) 2D Astigmatic Wavefront, and (C) Modified 2D Astigmatic Wavefront

\begin{tabular}{ccrrrrrrrc}
\hline & \multicolumn{1}{c}{ Samples } \\
\cline { 2 - 11 } Wavefront & $N_{4}$ & \multicolumn{1}{c}{$N_{5}$} & $N_{12}$ & $N_{13}$ & $N_{14}$ & $N_{24}$ & $N_{25}$ & $N_{26}$ & $N_{\text {else }}$ \\
\hline A & 7324 & 0 & 236 & 0 & 0 & 13 & 0 & 0 & 0 \\
B & 7324 & 1461 & 236 & 282 & 5 & 13 & 68 & 3 & 0 \\
C & 1500 & 1461 & 236 & 282 & 5 & 13 & 68 & 3 & 0 \\
\hline
\end{tabular}

$$
\begin{gathered}
W(\rho, \theta)=\sum_{j=0}^{p-1} c_{j} Z_{j}(\rho, \theta), \\
j=\frac{1}{2}(n(n+2)+m), \quad n=\left\lceil\frac{-3+\sqrt{9+8 j}}{2}\right], \\
m=2 j-n(n+2) ;
\end{gathered}
$$

$R_{n, m}(\rho)$

$$
=\sum_{s=0}^{(n-|m|) / 2} \frac{(-1)^{s}(n-s) !}{s ![0.5(n+|m|)-s] ![0.5(n-|m|)-s] !} \rho^{n-2 s},
$$

$$
\mathrm{N}_{n, m}=\left[\frac{2(n+1)}{1+\delta_{m 0}}\right]^{1 / 2}, \quad M_{m}(\theta)=\left\{\begin{array}{ll}
\cos (m \theta) & m \geqslant 0 \\
\sin (m \theta) & m<0
\end{array},\right.
$$

where $n$ and $m$ are the radial order and the azimuthal frequency, respectively, $p$ is the number of terms in the expansion, $c_{j}$ are the Zernike coefficients associated with their Zernike polynomial, and $[\cdot]$ denotes the ceiling (round-up) operator. A more convenient form to analyze the polynomial in Eq. (5) consists of rearranging the wavefront Zernike decomposition by grouping all the terms with the same radial degree and azimuthal frequency (Table 2):

$$
W(\rho, \theta)=\sum_{j=0}^{p-1} \gamma_{j} \rho^{n} M_{m}(\theta)=\sum_{j=0}^{p-1} \gamma_{j} G_{j}(\rho, \theta) .
$$

The transmittance function in polar coordinates can be expressed as $u_{0}(r, \theta)=\exp [i 2 \pi W(r, \theta) / \lambda]$. The general form of one term of the transmittance expansion, taking into account Eq. (7) with $r=r_{\text {pupil }} \rho$, is

$$
\exp \left(i \frac{2 \pi}{\lambda} W_{j}(r, \theta)\right)=\exp \left(i \frac{2 \pi}{\lambda} \gamma_{j} G_{j}\left(r_{\text {pupil }} \rho, \theta\right)\right) .
$$

Following the Nyquist criterion we can determine the correct sampling from the maxima of the spatial frequency both in $\rho$ and $\theta$ coordinates. Thus, expression (8) is correctly sampled provided that the total number of samples fulfills

$$
N_{j} \geqslant\left(\frac{n}{2^{n}} \delta_{m 0}+\pi|m|\right) \frac{4\left|\gamma_{j}\right|\left(2 r_{\text {pupil }}\right)^{n}}{\lambda},
$$

where $\delta_{m 0}$ is the Kronecker delta.

As stated above, we have used polar coordinates to avoid crossed terms. However, the sampling condition does not depend on the coordinate system used on the sampling pattern. Expression (9) coincides with the Nyquist condition for the 1D case in Eq. (4) considering $m=0$, i.e., radial symmetry. Again, highorder aberrations influencing the required number of samples is very small. If an optical system is described by a monochromatic wavefront $W(r, \theta)$ with small amounts of high order aberrations, from [4] and using Eq. (7), the estimate of the refractive power derived from the wavefront can be obtained as

$$
\begin{aligned}
\hat{F}(r, \theta) & =\left[W(r, \theta)+r(\partial W(r, \theta) / \partial r)^{-1}\right]^{-1} \\
& \cong r^{-1} \frac{\partial}{\partial r}\left[\sum_{j=3}^{p-1} \gamma_{j} r^{n} M_{m}(\theta)\right]=\sum_{j=3}^{p-1} n \gamma_{j} r^{n-2} M_{m}(\theta),
\end{aligned}
$$

where $n \gamma_{j}$ are directly related to the power vector components. We have removed the prismatic terms (i.e., for $|m|=1$ ) because the prism does not contribute

Table 2. Zernike Polynomials Decomposition and Rearranged Polynomials Decomposition

\begin{tabular}{ccccc}
\hline$j$ & $n$ & $m$ & $c_{n, m} Z_{n, m}(\rho, \theta)$ & $\gamma_{j} G_{j}(\rho, \theta)$ \\
\hline 0 & 0 & 0 & $c_{0,0}$ & $\left(c_{0,0}-\sqrt{3} c_{2,0}+\sqrt{5} c_{4,0}-\sqrt{7} c_{6,0}+\cdots\right)$ \\
1 & 1 & -1 & $2 c_{1,-1} \rho \sin (\theta)$ & $\left(2 c_{1,-1}-2 \sqrt{8} c_{3,-1}+3 \sqrt{12} c_{5,-1}-16 c_{7,-1}\right.$ \\
& & & $+\cdots) \rho \sin (\theta)$ \\
2 & 1 & 1 & $2 c_{1,1} \rho \cos (\theta)$ & $\left(2 c_{1,1}-2 \sqrt{8} c_{3,1}+3 \sqrt{12} c_{5,1}-16 c_{7,1}+\cdots\right) \rho \cos (\theta)$ \\
3 & 2 & -2 & $\sqrt{6} c_{2,-2} \rho^{2} \sin (2 \theta)$ & $\left(\sqrt{6} c_{2,-2}-3 \sqrt{10} c_{4,-2}+6 \sqrt{14} c_{6,-2}+\cdots\right) \rho^{2} \sin (2 \theta)$ \\
4 & 2 & 0 & $\sqrt{3} c_{2,0}\left(2 \rho^{2}-1\right)$ & $\left(2 \sqrt{3} c_{2,0}-6 \sqrt{5} c_{4,0}+12 \sqrt{7} c_{6,0}+\cdots\right) \rho^{2}$ \\
5 & 2 & 2 & $\sqrt{6} c_{2,2} \rho^{2} \cos (2 \theta)$ & $\left(\sqrt{6} c_{2,2}-3 \sqrt{10} c_{4,2}+6 \sqrt{14} c_{6,2}+\cdots\right) \rho^{2} \cos (2 \theta)$ \\
\hline
\end{tabular}


to the refractive power of an optical system. For the case $n=2(j=3, j=4$, and $j=5)$ we obtain $2 \gamma_{3}=-J_{45}$, $2 \gamma_{4}=-M=-1 / z_{c}$, and $2 \gamma_{5}=-J_{0}$, i.e., the $J_{45}, M$, and $J_{0}$ power vector components [4].

Let us consider that the initial transmittance function decomposition can be expressed as

$$
\begin{aligned}
u_{0}(r, \theta)= & \exp \left[i \frac{2 \pi}{\lambda} W(r, \theta)\right]=\exp \left[i \frac { 2 \pi } { \lambda } \left(\sum_{j=0}^{3} \gamma_{j} G_{j}(r, \theta)\right.\right. \\
& \left.\left.+\gamma_{4} r^{2}+\sum_{j=5}^{j_{\max }} \gamma_{j} G_{j}(r, \theta)\right)\right] \\
= & \exp \left[i \frac{2 \pi}{\lambda} W^{\prime}(r, \theta)\right] \exp \left[-\frac{i \pi}{\lambda z_{c}} r^{2}\right]
\end{aligned}
$$

If we add a divergent term of focus $z_{d}$ to the initial transmittance we obtain a new transmittance function (12) from which can be deduced the power vector component in Eq. (13):

$$
\begin{aligned}
& v_{0}(r, \theta)= u_{0}(r, \theta) \exp \left(\frac{i \pi}{\lambda z_{d}} r^{2}\right)=\exp \left[i \frac{2 \pi}{\lambda} W^{\prime}(r, \theta)\right] \\
& \times \exp \left[-\frac{i \pi}{\lambda}\left(\frac{1}{z_{c}}-\frac{1}{z_{d}}\right) r^{2}\right] \\
& \gamma_{4}^{\prime}=-\frac{M^{\prime}}{2}=-\frac{1}{2}\left(\frac{1}{z_{c}}-\frac{1}{z_{d}}\right)=\frac{z_{c}-z_{d}}{2 z_{d} z_{c}}
\end{aligned}
$$

Bearing in mind the relation between the $\gamma_{j}$ coefficients and the number of samples $N_{j}$ stated in Eq. (9), we can reduce the sampling requirements for

$$
\begin{aligned}
N_{4}^{\prime}<N_{4} \Leftrightarrow\left|\gamma_{4}^{\prime}\right|<\left|\gamma_{4}\right| \Leftrightarrow\left|\frac{z_{c}-z_{d}}{z_{d} z_{c}}\right| \\
<\left|-\frac{1}{z_{c}}\right| \Leftrightarrow z_{d}>\frac{z_{c}}{2} .
\end{aligned}
$$

Moreover, admitting an upper limit in the number of samples $N_{L}$ we can take advantage of the relation between $\gamma_{j}$ and the Nyquist criterion in Eq. (9) and obtain a limit for the $\gamma_{4 L}$ coefficient that allows us to calculate the propagated patterns with the number of samples $N_{L}$,

$$
\left|\gamma_{4 L}\right|=\frac{\lambda N_{L}}{2\left(2 r_{\text {pupil }}\right)^{2}} .
$$

We use Eq. (15) to compute how the original wavefront convergence must be modified, reducing the initial sampling condition, and we define an interval where the Nyquist condition is fulfilled,

$$
\gamma_{4 S}=\gamma_{4} \pm\left|\gamma_{4 L}\right|, \quad \Delta P=\left|M_{S}-M\right|=\frac{\lambda N_{L}}{\left(2 r_{\text {pupil }}\right)^{2}},
$$

where $2 \gamma_{4 S}$ is the fourth power vector component of the spherical wave to subtract from the original wavefront.
Let us consider a spherical wavefront of curvature radius $z_{c}=15.75 \mathrm{~mm}, \lambda=555 \mathrm{~nm}$, and a window width $2 r_{\text {pupil }}=8 \mathrm{~mm}$. The $G_{j}$ polynomials decomposition and Eq. (9) provide the required number of samples by each polynomial in order to fulfill the Nyquist condition. Table 1(A) shows the sampling condition for each $G_{j}$ up to $j=35$ th (order seventh). Note that the fourth polynomial samples number, corresponding to defocus aberration, is almost 2 magnitude orders higher than the 12th one, corresponding to spherical aberration. Hence the fourth term of the wavefront can be modified to reduce the sampling requirements adding a divergent phase to the original one. This procedure of eliminating the convergence factor is quite common in ocular wavefront analyzers, and it is usually named as removing the reference sphere [5].

In the case of a wavefront affected by astigmatism, the Nyquist condition of the function can be further reduced up to the sampling imposed by astigmatic aberration. Table 1(B) shows the sampling requirements for each polynomial decomposition of a $2 \mathrm{D}$ astigmatic wavefront convergent to $z_{c}=15.75$ and $2 r_{\text {pupil }}=8 \mathrm{~mm}$. The condition imposed by the fifth polynomial is around 1500 samples, so this is the number that limits the sampling. In Table 1(C), we show the sampling requirements resulting from taking $N_{L}=1500$. From Eq. (16), it is possible to obtain the propagated patterns correctly sampled at $\Delta P$ $=13.4 \mathrm{D}$ around the convergence distance. Finally, as a direct application of the method, notice that from Eq. (1) we can combine the convergent factor that is carried by the input beam with the divergent one from the propagation kernel. Thus, if we identify the propagation distance $z$ with the divergent focus $z_{d}$, conditions for correct Fresnel sampling are those derived from Eq. (12). Therefore, an efficient algorithm for calculating the Fresnel pattern around the focus will consist of taking the input beam, canceling part of the phase factor with a divergent beam, and then performing an FFT of the result. Notice that, from Eq. (16), the number of samples needed for correct reconstruction of the beam decreases dramatically as we approach the focus.

This work has been supported by the Spanish Ministerio de Educación y Ciencia project FIS200505053.

\section{References}

1. D. Mas, J. Espinosa, J. Pérez, and C. Illueca, J. Mod. Opt. 53, 259 (2006).

2. D. Mas, J. Pérez, C. Hernández, C. Vázquez, J. J. Miret, and C. Illueca, Opt. Commun. 227, 245 (2003).

3. B. M. Hennelly and J. T. Sheridan, J. Opt. Soc. Am. A 22, 917 (2005).

4. R. Iskander, R. A. Davis, M. J. Collins, and R. Franklin, Ophthalmic Physiol. Opt. 27, 245 (2007).

5. L. Lundström, S. Manzanera, P. M. Prieto, D. B. Ayala, N. Gorceix, J. Gustafsson, P. Unsbo, and P. Artal, Opt. Express 15, 12654 (2007). 